\title{
Divergence and Vorticity from Aircraft Air Motion Measurements
}

\author{
DONALD H. LENSCHOW \\ National Center for Atmospheric Research,* Boulder, Colorado \\ Verica Savic-Jovcic And Bjorn Stevens \\ Department of Atmospheric and Oceanic Sciences, University of California, Los Angeles, Los Angeles, California
}

(Manuscript received 16 November 2006, in final form 29 March 2007)

\begin{abstract}
This paper considers the accuracy of divergence estimates obtained from aircraft measurements of the horizontal velocity field and points out an error that appears in these estimates that has heretofore not been addressed. A procedure for eliminating this error is presented. The divergence and vorticity are estimated from the coefficients of a least squares fit to a wind field obtained from the Second Dynamics and Chemistry of Marine Stratocumulus (DYCOMS-II) circular flight legs. These estimates are compared with estimates from numerical models and satellites and with airplane estimates based on tracer budgets and the temporal changes in cloud-top height. The estimates are consistent with expectations and estimates using other methods, albeit somewhat high. Furthermore, significant differences occur among the cases, likely due to the large differences in the techniques. The results indicate that the wind field technique is a viable approach for estimating mesoscale divergence if the wind measurements are accurate. The largest source of wind field systematic error may be the result of flow distortion effects on the air velocity measurement and limitations of in-flight calibrations. Because of flow distortion, the only way the current systems can be calibrated is by flight maneuvers, which assume a steady-state homogeneous nonturbulent atmosphere. Analysis of the errors in this technique suggests that wind field measurements with minimal systematic errors should provide estimates of divergence with much greater accuracy than is now possible with other existing methods.
\end{abstract}

\section{Introduction}

Measuring mean vertical air velocity $\bar{w}$ from aircraft has been a long-sought goal of observationalists because of its crucial role in modulating important processes in the atmosphere. Areas of generally rising air, for example, have enhanced cumulus convection and often have an indistinct boundary layer top, while areas of downward-moving air suppress deep convection and generally have a well-defined boundary layer top due, in part, to adiabatic warming.

Since the magnitude of $\bar{w}$ in quiescent regions is generally less than $0.01 \mathrm{~m} \mathrm{~s}^{-1}$, it is too small to be measured

\footnotetext{
* The National Center for Atmospheric Research is sponsored by the National Science Foundation.
}

Corresponding author address: Donald H. Lenschow, MMM Division, NCAR, P.O. Box 3000, Boulder, CO 80307-3000.

E-mail: lenschow@ucar.edu directly by current airborne air motion sensing technology. Currently, aircraft systems are capable only of measuring fluctuations in $w$ as there is no way to obtain an absolute calibration for estimating $\bar{w}$. This is a formidable barrier, since even with the development of, for example, a Doppler laser system that could, in principle, measure an absolute value of the radial air velocity via digital counting techniques at a location far removed from the flow-distorted region close to the aircraft, the required angular accuracy of the laser beam orientation would likely preclude the possibility of attaining such accuracy. However, an alternative approach is to estimate a mean divergence $\bar{D}$, which through continuity and the assumption of incompressibility, is related to $\bar{w}$ by

$$
\frac{\partial \bar{w}}{\partial z}=-\frac{\partial \bar{u}}{\partial x}-\frac{\partial \bar{v}}{\partial y}=-\bar{D},
$$

where $\bar{u}$ and $\bar{v}$ are the mean east and north wind components, respectively.

DOI: 10.1175/2007JTECHA940.1

(C) 2007 American Meteorological Society 
Similarly, the mean vertical vorticity component $\bar{\zeta}$ can be obtained from the averaged velocity derivatives,

$$
\bar{\zeta}=\frac{\partial \bar{v}}{\partial x}-\frac{\partial \bar{u}}{\partial y} .
$$

Applications and measurements of $\bar{\zeta}$ and its relation to $\bar{D}$ are discussed by Lenschow et al. (1999, hereafter LKS).

Previous measurements of $\bar{D}$ and $\bar{\zeta}$ have been obtained mostly from rawindsonde sounding networks. Davies-Jones (1993) summarizes the use of formulas applied to a polygonal array of stations that had been developed and applied for more than $40 \mathrm{yr}$. In addition, Doppler radars have also been used to measure mesoscale $\bar{D}$, especially in the Tropics (e.g., Mapes and Lin 2005; Mapes and Houze 1995). However, they require the presence of sufficient scatterers, such as precipitation particles and assumptions about the free-fall velocities of the scatterers. Angevine (1997) used a triangle of $915-\mathrm{MHz}$ wind profilers 5.5 to $7 \mathrm{~km}$ on a side to estimate divergence from 120-m height through the top of the planetary boundary layer (PBL). Gultepe et al. (1990) reported on concurrent measurements of $\bar{D}$ using a circular aircraft flight track and a ground-based Doppler lidar. Although results from the two approaches were in reasonable agreement, the minimum detectable signal level was larger than required for most studies requiring divergence measurements. Brost et al. (1982) also tried to estimate $\bar{D}$ using aircraft measurements but used only one component of the wind field.

Recently, this topic has been revisited by LKS, who show that with accurate horizontal air velocity measurements, it should be possible to estimate divergence and vorticity in the boundary layer with accuracy acceptable for many observational studies of meso- and larger-scale dynamics. They also discuss the sources of both random and systematic errors in measuring divergence and vorticity and strategies to optimize the measurement accuracy. The results of this work, which included some preliminary measurements obtained from the National Center for Atmospheric Research (NCAR) C-130 aircraft during the Aerosol Characterization Experiment (ACE-1), were sufficiently encouraging that a flight pattern was included in the Second Dynamics and Chemistry of Marine Stratocumulus (DYCOMS-II) field campaign (Stevens et al. 2003b) that would allow measurement of divergence and vorticity on a routine basis for most of the flights.

Here we extend the discussion of airborne divergence and vorticity measurement, pointing out further issues in obtaining the measurements from current air- craft sensing systems and using data collected in DYCOMS-II as a basis for comparison with predicted measurement accuracy. Our focus is on estimates of $\bar{D}$, which are compared to estimates from satellite products and with the reanalysis of meteorological data provided by the European Centre for Medium-Range Weather Forecasts, as well as estimates inferred from other DYCOMS-II measurements. The manuscript is organized as follows. In section 2, we review the measurement method of LKS and propose a new method that offers some advantages over the line-integral approach. In section 3 we quantify sources of error. Section 4 discusses our results, and we conclude with a brief summary in section 5 .

\section{Divergence and vorticity estimate methods}

\section{a. Line-integral method}

LKS applied the divergence and Stokes theorems to estimate $\bar{D}$ and $\bar{\zeta}$ from closed integrals of, respectively, the horizontal velocity component normal $v_{\perp}$ and tangent $v_{\|}$to each element of the track $d l$ enclosing the area $A$ :

$$
\bar{D}=\frac{1}{A} \oint_{C} v_{\perp} d l
$$

and

$$
\bar{\zeta}=\frac{1}{A} \oint_{C} v_{\|} d l .
$$

They noted that the most efficient flight track is a circle because 1) it has the largest enclosed area of any closed curve of a given circumference and 2) the rate of turning is slow enough for a circle taking more than a few tens of minutes to complete so that there should be no significant increase in wind measurement error compared to a straight flight path. A circle is also optimal for measuring the mean wind, that is, the average of the wind vector around the entire circle, since, as we will show, many of the most significant measurement errors cancel out when averaged over a circle. Polygonal tracks incur such rapid turn rates at the vertices that the measurement accuracy is degraded. The LKS approach was to fly constant rate turns so that, ideally, in a Lagrangian framework (i.e., the coordinate system drifting with the mean wind) the aircraft track would be a circle.

The LKS technique is well suited to perfectly executed closed flight paths in a stationary wind field. However, in the real world the flight tracks are neither exactly circular nor closed, and the wind field will likely 

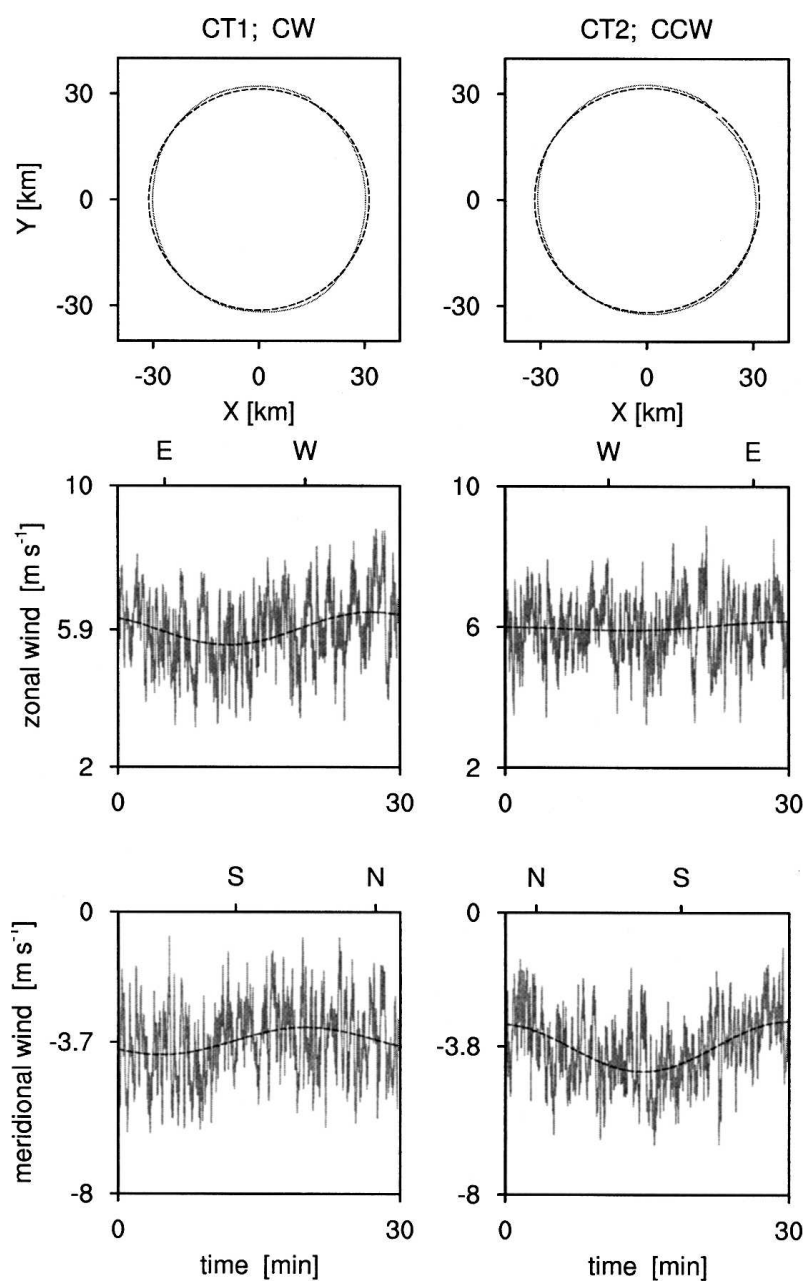

FIG. 1. An example of zonal and meridional winds from two successive circular flight paths during RF07 (24 Jul 2001) at about 700-m altitude in DYCOMS-II. Solid lines are the aircraft track, and dashed lines are circles in the top panels and best-fit sinusoids to the wind measurements in the lower panels.

not be stationary. A typical example of actual circular flight paths from DYCOMS-II and the measured $u$ and $v$ wind components is shown in Fig. 1. LKS dealt with the closure issue by integrating around the circle to the point of minimum distance from the starting point, but this still means that an error may exist that depends on the magnitude of this distance. They did not deal with the possibility of a nonstationary wind field.

\section{b. Regression method}

To address these shortcomings, we have developed a method that requires neither a closed nor a circular flight path and corrects for linear changes in the wind field with time. To start, we expand the wind vector via a Taylor series from a reference point near the center of the flight track. The mean wind velocity over the track is $\boldsymbol{v}_{0}$. For convenience, we adopt a Lagrangian coordinate system with the reference point advected with the mean wind, since the aircraft flight track is circular in this coordinate system. Considering only the first-order terms,

$$
\boldsymbol{v}=\boldsymbol{v}_{0}+\frac{\partial \boldsymbol{v}}{\partial x} \Delta x+\frac{\partial \boldsymbol{v}}{\partial y} \Delta y+\frac{\partial \boldsymbol{v}}{\partial t} \Delta t,
$$

where $\Delta x$ and $\Delta y$ are the eastward and the northward displacements from the reference point and $\Delta t$ is the elapsed time from the beginning of the flight leg.

Although the method works for any arbitrary flight track that traverses a sufficient area to allow estimates of horizontal gradients, we apply it here, both for illustration and because the DYCOMS-II flight tracks (advected with the mean wind) are nearly circular, to a perfect circle of radius $R$. The displacements $\Delta x$ and $\Delta y$ then are simply $\Delta x=R \sin \Psi$ and $\Delta y=R \cos \Psi$, where $\Psi$ is the azimuth angle of the airplane flight track. Therefore, expanding (5) for both the easterly $u$ and the northerly $v$ wind components,

$$
\begin{gathered}
u=u_{0}+\frac{\partial u}{\partial x} R \sin \Psi+\frac{\partial u}{\partial y} R \cos \Psi+\frac{\partial u}{\partial t} \Delta t \quad \text { and } \\
v=v_{0}+\frac{\partial v}{\partial x} R \sin \Psi+\frac{\partial v}{\partial y} R \cos \Psi+\frac{\partial v}{\partial t} \Delta t .
\end{gathered}
$$

A schematic illustration of this method is shown in Fig. 2, where a circular flight path is embedded in an easterly flow with a spatial gradient $\left[u=u_{0}+(\partial u / \partial x) \Delta x\right]$. The measured wind in this idealized case is also depicted in the figure and has the form $u=u_{0}+a \sin \Psi$. Therefore,

$$
\bar{D}=\frac{\partial u}{\partial x}=\frac{a}{R} .
$$

In practice, the airplane is flown at an approximately constant turn rate $\omega$, so that $\Psi=\omega \Delta t+\Psi_{0}$, where $\Psi_{0}$ is the initial azimuth angle of the flight track. We can then estimate $R$, $\omega$, and $\Psi_{0}$ by least squares fits to the aircraft flight path in a coordinate system that is allowed to advect with the mean wind. We then substitute these values into (6) and (7) in order to estimate $\bar{D}$ and $\bar{\zeta}$ by a least squares fit to the aircraft wind measurements.

\section{Measurement system errors}

\section{a. Systematic errors}

In addition to random errors due to turbulence, there are significant systematic errors introduced into the air- 

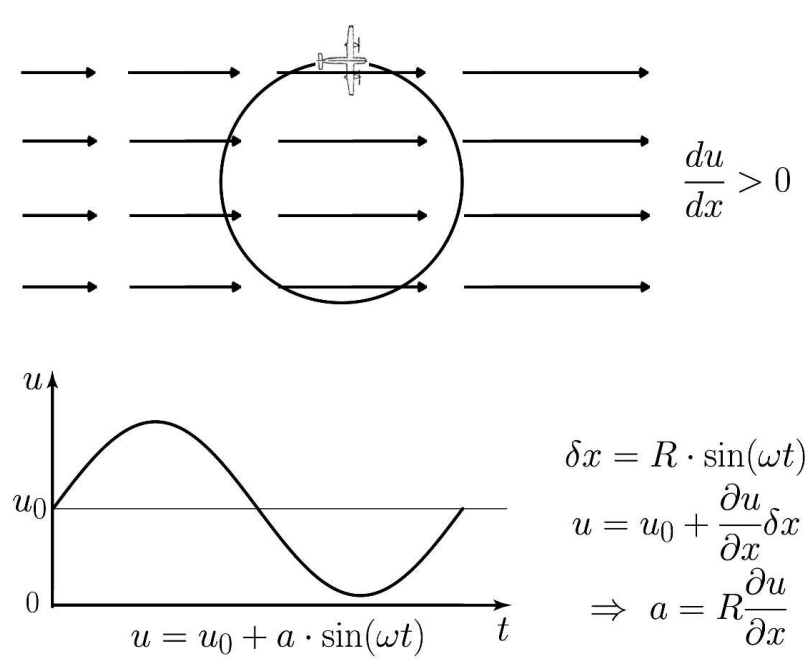

$$
\begin{aligned}
& u=u_{0}+\frac{\partial u}{\partial x} \delta x+\frac{\partial u}{\partial y} \delta y+\frac{\partial u}{\partial t} \delta t \\
& u=u_{0}+a_{x} \cdot \sin (\omega t)+a_{y} \cdot \cos (\omega t)+a_{t} \cdot t
\end{aligned}
$$

FIG. 2. A schematic illustration of how the regression method is applied to the wind components on the circular flight tracks.

craft-measured wind field by the measurement system. These errors are critical for estimating divergence and vorticity. The technique used for measuring winds from aircraft is described by Lenschow (1986). The three attitude angles and coordinates are shown in Fig. 3. Basically, the wind vector $\boldsymbol{v}$ is obtained by subtracting the velocity of the air relative to the aircraft $\boldsymbol{v}_{\text {ap }}$ from the velocity of the aircraft relative to the earth $\boldsymbol{v}_{\mathrm{pe}}$. Here, $\boldsymbol{v}_{\mathrm{pe}}$ includes also the mean wind advection. The velocity $\boldsymbol{v}_{\mathrm{pe}}$ is obtained from an inertial reference system (IRS), while $\boldsymbol{v}_{\text {ap }}$ is obtained from pressure difference measurements between ports on a nose-mounted radome. Since the IRS measures in an earth-referenced coordinate system and $\boldsymbol{v}_{\text {ap }}$ is measured relative to the aircraft, it is necessary to rotate $\boldsymbol{v}_{\text {ap }}$ to an earth-referenced coordinate system before calculating $\boldsymbol{v}$. The angular orientation or attitude angles of the aircraft are obtained from the IRS, which continuously calculates its angular orientation with respect to the local earth coordinates.

The velocity $\boldsymbol{v}_{\text {ap }}$ is obtained from measurements of the true airspeed $U_{a}$, the attack angle $\alpha$, and the sideslip angle $\beta$. The true airspeed is obtained from the Pitotstatic pressure difference, with a correction for air den-
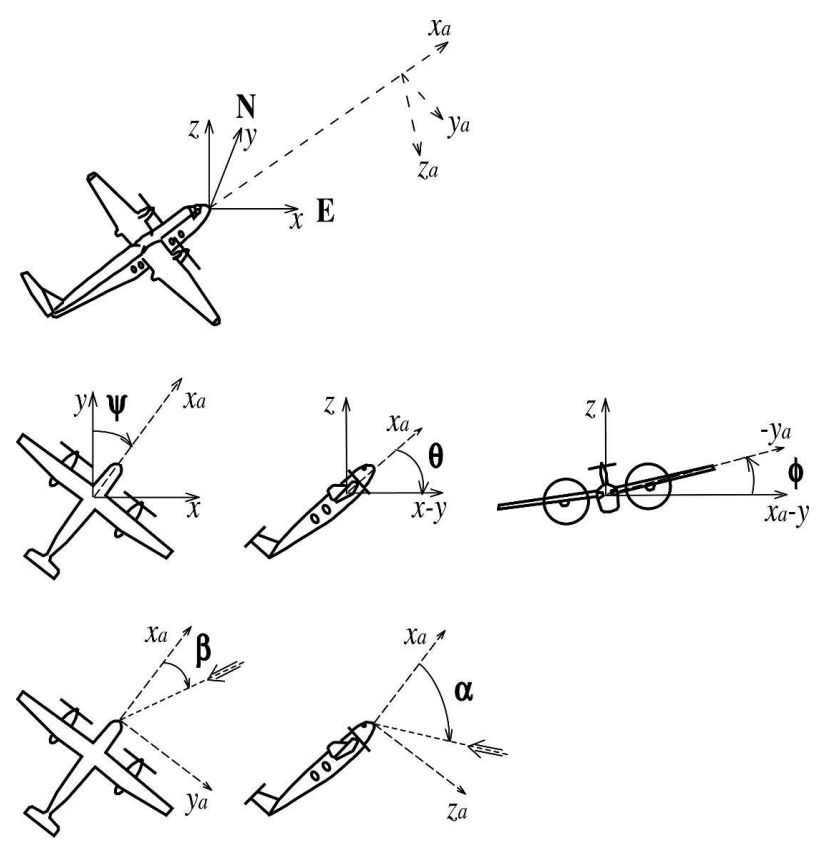

FIG. 3. Schematic showing the attitude angles and axes used to define the airplane coordinate systems. Here $\psi, \theta$, and $\phi$ are the heading, pitch, and roll attitude angles, respectively; and $\alpha$ and $\beta$ are the attack and sideslip airflow angles, respectively.

sity. Here $\alpha$ and $\beta$ are obtained from pressure differences across ports on the radome, also corrected for air density. The $\alpha$ ports are located in the vertical plane of the aircraft roughly at $\pm 40^{\circ}$ on either side of the longitudinal airplane axis and the $\beta$ ports roughly at $\pm 40^{\circ}$ in the horizontal plane of the aircraft. Attack angle $\alpha$ is positive downward and $\beta$ is positive rotated clockwise looking down from above.

The attitude angles measured by the IRS are defined in the following sequence: first, the roll angle $\phi$ is a rotation about the longitudinal axis, positive with the left wing (looking forward) lifted; second, the pitch angle $\theta$ is a rotation about the lateral axis, positive with the nose lifted; and third, the true heading angle $\psi$ is a rotation about the local earth vertical axis, positive for clockwise rotation looking down, starting from the north.

There are additional terms in the air velocity equations if $\boldsymbol{v}_{\text {ap }}$ is not measured at the same point as $\boldsymbol{v}_{\mathrm{pe}}$. (Lenschow 1986). However, these are generally small and in any case are not important for measuring $\bar{D}$ and $\bar{\zeta}$. The final form of the air velocity equations used here, then is

$$
\begin{aligned}
& u=-U_{a} D_{a}^{-1}[\sin \psi \cos \theta+\tan \beta(\cos \psi \cos \phi+\sin \psi \sin \theta \sin \phi)-\tan \alpha(\cos \psi \sin \phi-\sin \psi \sin \theta \cos \phi)]+u_{\mathrm{pe}} \\
& v=-U_{a} D_{a}^{-1}[\cos \psi \cos \theta-\tan \beta(\sin \psi \cos \phi-\cos \psi \sin \theta \sin \phi)+\tan \alpha(\sin \psi \sin \phi+\cos \psi \sin \theta \cos \phi)]+v_{\mathrm{pe}}
\end{aligned}
$$




$$
\begin{aligned}
w= & -U_{a} D_{a}^{-1}[\sin \theta-\tan \beta \cos \theta \sin \phi-\tan \alpha \cos \theta \cos \phi] \\
& +w_{\mathrm{pe}} .
\end{aligned}
$$

Here, $D_{a}=\sqrt{1+\tan ^{2} \alpha+\tan ^{2} \beta}$ is a correction for the fact that the airspeed Pitot-static pressure measures approximately the magnitude of the airspeed independent of the direction of flow.

For convenience in analyzing measurements of $\bar{D}$ and $\bar{\zeta}$, as well as errors in the air velocity measurement, we rotate $\{u, v\}$ to a coordinate system whose directions are defined by the local horizontal plane and $\psi$ :

$$
\begin{aligned}
& v_{\perp}=u \cos \psi-v \sin \psi \quad \text { and } \\
& v_{\|}=u \sin \psi+v \cos \psi .
\end{aligned}
$$

These we refer to as the horizontal velocity components normal to and parallel to the aircraft heading. Carrying out the rotation we obtain

$$
\begin{aligned}
v_{\perp}= & -U_{a} D_{a}^{-1}(\tan \beta \cos \phi-\tan \alpha \sin \phi)+u_{\mathrm{pe}} \cos \psi \\
& -v_{\mathrm{pe}} \sin \psi \text { and } \\
v_{\|}= & -U_{a} D_{a}^{-1}(\cos \theta+\tan \beta \sin \theta \sin \phi+\tan \alpha \sin \theta \cos \phi) \\
& +u_{\mathrm{pe}} \sin \psi+v_{\mathrm{pe}} \cos \psi
\end{aligned}
$$

These equations are the basis for an error propagation analysis, which we apply to the circular flight track used here to measure $\bar{D}$ and $\bar{\zeta}$. Previous analyses considered only errors in air velocity measurement in straight and level flight. It turns out that the circular track introduces a new error that can be significant for divergence measurements, which is not present for straight and level flight.

Before further evaluating how various terms contribute to measurement error, we first review the standard procedures the NCAR Research Aviation Facility (RAF) uses for calibrating the wind field measurements. We assume that the IRS accurately measures the airplane velocity (with global positioning system updating) and attitude angles. But because of flow distortion effects, sensor inaccuracies, and asymmetries in the nose geometry and the sensing ports, the airflow sensor outputs are calibrated by a series of in-flight maneuvers (Lenschow 1986; Kalogiros and Wang 2002). In this analysis, $\alpha$ and $\beta$ are calculated as

$$
\alpha=\delta \alpha+c_{\alpha} \frac{\Delta_{\alpha} p}{q} \text { and } \beta=\delta \beta+c_{\beta} \frac{\Delta_{\beta} p}{q},
$$

where $\delta \alpha$ and $\delta \beta$ are measured biases in the attack and sideslip angles; $q$ is the dynamic pressure measured by a Pitot-static pressure difference; and $c_{\alpha}$ and $c_{\beta}$ are sen- sitivity coefficients that linearly relate the attack and sideslip angles to pressure differences across the vertical and horizontal radome pressure ports, respectively.

The RAF uses the following flight maneuvers to calibrate the measurement of $\alpha$.

1) A sinusoidal variation in airspeed while the airplane altitude and heading are held constant. This modulates $\theta$ and $\alpha$, which allows us to estimate $c_{\alpha}$.

2) A flight leg with speed, altitude, and heading held constant; therefore, $\phi \simeq 0$.

We then assume the mean vertical air velocity is zero over the flight leg so that from (11), $\sin \theta \simeq \tan \alpha \cos \theta$. Thus, the condition $\bar{w}=0$ in straight and level flight is equivalent to $\tan (\theta-\alpha)=\theta-\alpha=0$. This allows us to either add a constant to $\theta$ or adjust $\delta \alpha$ so as to zero the vertical velocity. While this does not uniquely determine $\delta \alpha$, (11) shows that, from the perspective of the estimate of $w$, it is only necessary to constrain the difference between $\theta$ and $\alpha$. Of course, if the real vertical air velocity is not zero, there will be a bias in the $\alpha$ measurement.

Similarly, to calibrate $\beta$ the RAF uses 1) a sinusoidal variation in heading, which also modulates $\beta$, and thus $c_{\beta}$ is adjusted so as to eliminate any sinusoidal variation that may exist in $v_{\perp}$; and 2) a pair of 2-min legs on opposite headings in quiescent air where a constant wind field is assumed, from which $\delta \beta$ (and calibration factors for $U_{a}$ ) are chosen so as to eliminate the differences in both $\bar{u}$ and $\bar{v}$.

As shown by LKS, the above method does not determine $\delta \beta$ with sufficient absolute accuracy to determine $\bar{D}$, and so a standard procedure for reducing the error in $\bar{D}$ is to fly circles both clockwise (CW) and counterclockwise (CCW). By taking the average of $\bar{D}$ and $\bar{\zeta}$ for both CW and CCW circles, errors in $v_{\perp}$ and $v_{\|}$ that are modulated by $\psi$ will cancel out when integrated around the circle. This is similar to the opposite headings procedure discussed above for calibrating mean horizontal air velocity components $\bar{u}$ and $\bar{v}$ but is a significantly more accurate procedure for estimating $\delta \beta$ since it uses the entire set of the circular flight tracks. This procedure is estimated to be accurate to about $0.01^{\circ}$ in $\beta$ or $v_{\perp} \simeq 0.02 \mathrm{~m} \mathrm{~s}^{-1}$, with the limitation imposed primarily by real variability in the wind field.

Unfortunately, when flying $\mathrm{CW}$ and $\mathrm{CCW}$ circles, we also introduce a roll angle to maintain the turn in contrast to straight flight legs where $\phi \simeq 0$. This roll angle is

$$
\phi= \begin{cases}\Delta \phi & \text { positive for } \mathrm{CW} \text { rotation } \\ \Delta \phi & \text { negative for CCW rotation. }\end{cases}
$$


If we assume that the centripetal acceleration while flying around the circle is balanced by the horizontal component of lift normal to the trajectory resulting from banking the airplane, the resulting roll angle is

$$
\Delta \phi= \pm \arctan \frac{2 \pi U_{a}}{T g},
$$

where $T$ is the flight duration around the circle and $g$ is gravity. For a 30 -min circle at $100 \mathrm{~m} \mathrm{~s}^{-1}, \Delta \phi \simeq \pm 2^{\circ}$.

This constant roll angle introduced by circular flight legs results in our having to consider other terms as possible error sources that have not previously been important for analysis of straight and level flight legs. As discussed above, for straight flight legs, it is not necessary to distinguish between offsets in $\alpha$ and offsets in $\theta$, because the two angles appear approximately as a term involving $\tan (\theta-\alpha)$. However, for $v_{\perp}$ in circular flight, there is a difference in that there is a term in $v_{\perp}$ that contains the attack angle, $\tan \alpha \sin \phi$, while there is no term involving $\theta$. Hence the absolute attack needs to be determined.

To make these points more quantitatively, we use (14) and (15) to carry out an analysis of the systematic errors in $\bar{D}$ and $\bar{\zeta}$ due to biases in $\alpha$ and $\beta$, considering the possibility of a nonzero roll angle. We denote such biases by $\delta \alpha$ and $\delta \beta$ for attack and sideslip, respectively, and assume that they are small enough (typically $<6^{\circ}$ ) that $\tan (\alpha+\delta \alpha) \simeq \tan \alpha+\delta \alpha$ [and similarly for $\tan (\beta$ $+\delta \beta)]$. We also assume that the flight path (which is advected with respect to earth-based coordinates at the mean wind speed) is close enough to a circle that we can assume a circular flight path for our error analysis. We introduce $\delta \alpha$ and $\delta \beta$ into (14) and (15) and assume that the true $\bar{D}=\bar{\zeta}=0$. The errors in $\bar{D}$ and $\bar{\zeta}$ are obtained by flying circles starting at the same angular location on the circle for both the $\mathrm{CW}$ and $\mathrm{CCW}$ directions. If we arbitrarily select zero heading (pointing north) for the $\mathrm{CW}$ circle and $-\pi \operatorname{rad}$ (pointing south) for the CCW circle, the errors are

$$
\begin{aligned}
& \delta \bar{D}=\frac{R}{2 A}\left[\int_{0}^{2 \pi} v_{\perp}^{(\delta)}(\mathrm{CW}) d \Psi+\int_{-\pi}^{-3 \pi} v_{\perp}^{(\delta)}(\mathrm{CCW}) d \Psi\right] \text { and } \\
& \delta \bar{\zeta}=\frac{R}{2 A}\left[\int_{0}^{2 \pi} v_{\|}^{(\delta)}(\mathrm{CW}) d \Psi+\int_{-\pi}^{-3 \pi} v_{\|}^{(\delta)}(\mathrm{CCW}) d \Psi\right]
\end{aligned}
$$

where

$$
\begin{aligned}
& v_{\perp}^{(\delta)}=-U_{a} D_{a}^{-1}[\tan (\beta+\delta \beta) \cos \Delta \phi-\tan (\alpha+\delta \alpha) \sin ( \pm \Delta \phi)]+u_{\mathrm{ap}} \cos \psi-v_{\mathrm{ap}} \sin \psi \quad \text { and } \\
& v_{\|}^{(\delta)}=-U_{a} D_{a}^{-1}[\cos \theta+\tan (\beta+\delta \beta) \sin \theta \sin ( \pm \Delta \phi)+\tan (\alpha+\delta \alpha) \sin \theta \cos \Delta \phi]+u_{\mathrm{ap}} \sin \psi+v_{\mathrm{ap}} \cos \psi
\end{aligned}
$$

For small angles (18) and (19) reduce to

$$
\begin{aligned}
\delta \bar{D} & \simeq \frac{2 U_{a} D_{a}^{-1}}{R} \delta \alpha \Delta \phi \text { and } \\
\delta \bar{\zeta} & \simeq \frac{2 U_{a} D_{a}^{-1} \sin \theta}{R} \delta \beta \Delta \phi .
\end{aligned}
$$

These errors result from the terms in (14) and (15) involving $\sin \phi$, which change sign depending on whether the circle is flown CW or CCW. From the point of view of $v_{\perp}$ or $\bar{D}$, a bias in $\alpha$ is transformed into an error in $v_{\perp}$, which does not cancel out when the airplane is flown in the opposite direction around the circle. Similarly, a bias in $\beta$ is transformed into an error in $v_{\|}$, which does not cancel out when the airplane is flown in opposite directions; however, in this case the additional factor of $\sin \theta$ leads to negligible errors in $\bar{\zeta}$ even if $\delta \beta$ is several degrees. Finally, these biases make no appreciable contribution to the means of $u$ and $v$ integrated around the circles.

Equation (22) highlights the significant contribution that small biases in the estimate of absolute attack can have on estimates of $\bar{D}$. For instance, for an error of only $1^{\circ}$ in $\alpha$, this term contributes $0.06 \mathrm{~m} \mathrm{~s}^{-1}$ to $v_{\perp}$, which corresponds to $\delta \bar{D} \simeq 4 \times 10^{-6} \mathrm{~s}^{-1}$, irrespective of which direction the circle is flown. So even a $1^{\circ}$ bias in the attack can contribute to an error in $\bar{D}$ that is as large as the signal we are trying to measure.

The impact of small biases in $\alpha$ was not previously appreciated and highlights the need to accurately estimate the absolute angle of attack. To the extent that one is interested only in measurements of the mean wind in straight and level flight, it makes no difference whether the constraint of $\bar{w}=0$ is met by introducing an offset in either $\theta$ or $\alpha$, and in the past the RAF has introduced a constant offset in $\theta$ to satisfy this constraint. This procedure, which amounts to realigning the IRS coordinate system, is problematic for a turning aircraft for which the absolute value of attack becomes important. However, because $\bar{w}$ is the component of the flow perpendicular to the geopotential and the reference for $\theta$ is the geopotential, which is continuously measured very accurately by the IRS, by not incorpo- 
rating any offset in $\theta$, and choosing $\delta \alpha$ such that $\bar{w}=0$, the $\alpha$ measurement will be aligned to the local horizontal, thus defining an absolute $\alpha$.

Of course $\bar{w}$ is not expected to completely vanish over the flight leg, and such a procedure does not account for the possibility that flow distortion effects on $\alpha$ are modulated by $\theta$ and $U_{a}$. While we are unable to quantify the latter, the error contributed by $\bar{w} \neq 0$ can be estimated as follows. If we assume, for example, that $\bar{w}=10^{-2} \mathrm{~m} \mathrm{~s}^{-1}$, this leads to an angular error of $10^{-4}$ $\mathrm{rad}$ and, for a 30 -min circle at $100 \mathrm{~m} \mathrm{~s}^{-1}$, from (22) $\delta \bar{D} \approx 5 \times 10^{-8} \mathrm{~s}^{-1}$, which is negligible compared to typical values of $\bar{D}$.

A simple estimate of the effects of flow distortion, transducer drift, etc., on $\alpha$ can be obtained by looking at how much $\delta \alpha$ varies from leg to leg (provided individual legs are long enough for $\bar{w}$ to vanish) or flight to flight. That is, variations in $\delta \alpha$ among the flights (or legs) can be used as a rough indicator of how accurately one can align $\alpha$ to the local horizontal. The mean standard deviation of $\delta \alpha$ over all the legs within each flight is about $6 \times 10^{-3} \mathrm{rad}\left(0.33^{\circ}\right)$, while the mean standard deviation of $\delta \alpha$ over all the analyzed flights is about $10^{-3} \mathrm{rad}\left(0.06^{\circ}\right)$, which amounts to an error in $w$ of $\sim 0.1$ $\mathrm{m} \mathrm{s}^{-1}$. The former we consider an upper bound because variations in $\delta \alpha$ may occur during a flight due to systematic changes in $\theta$ associated with the changing airplane mass (which decreases through the flight due to fuel burnoff), inducing systematic flow distortion changes. These effects would mostly cancel out in the flight-to-flight standard deviation estimate.

From (22), $\delta \bar{D} \simeq 2.4 \times 10^{-7} \mathrm{~s}^{-1}$, which is small compared to expected values of $\bar{D}$. However, this is larger than what would be expected due solely to violation of the assumption of $\bar{w}=0$, which implies that other factors, such as flow distortion effects and random measurement errors, may also contribute.

As noted earlier, the measurement of $\bar{D}$ on $\mathrm{CW}_{-}$ CCW circular flight paths provides an extremely accurate basis for estimating $\delta \beta$. Half the difference between the average $\bar{D}$ for all the $\mathrm{CW}$ circles and for all the CCW circles considered here using the RAF data was $8.37 \times 10^{-6} \mathrm{~s}^{-1}$. From (18), this gives $\delta \beta \simeq 2.4 \times$ $10^{-3} \mathrm{rad}\left(0.14^{\circ}\right)$, which gives an average error in $v_{\perp}$ over all the flights considered here of $\simeq 0.24 \mathrm{~m} \mathrm{~s}^{-1}$. This error is considerably larger than our estimate of the achievable accuracy using the circular flight paths, and the result suggests that the circular flight path approach would be a preferred technique for calibrating the $\beta$ measurement.

The above procedure of determining offsets in $\alpha$ and $\beta$ from the flight data rather than by some static calibration or flow modeling calculations is a result of both flow distortion effects and sensor inaccuracies and actual angular misalignments between the air velocity sensing system and the IRS coordinate system. The airplane nose is neither perfectly symmetrical nor free of surface irregularities, and thus the alignment of the air sensing ports on the surface of the nose relative to the IRS axes is not absolute. In-flight calibration maneuvers cannot remove all these systematic errors, since the real wind field varies in time and space.

Any errors that remain from these residual misalignments between the IRS coordinates and the gust probe can be estimated as follows. Denoting a misalignment 1) about the IRS $x$ axis, $\xi ; 2$ ) about the $y$ axis, $\eta$; and 3) about the $z$ axis, $\nu$, and assuming that this remaining misalignment is small enough that we can neglect the effects of the order of the rotation, then the terms involving the cosine of the angles approach unity while terms involving the product of two sines become negligible. (Note that this is different from the analysis where we modulate the wind components by varying the heading angle; here the misalignment angles are constant with respect to the turning of the aircraft. That is why we can use small angle approximations here.) Then, using matrix notation, the air velocity vector in the airplane coordinate system assuming accurate determination of the angles between the gust probe and the IRS, with respect to the air velocity vector that is misaligned with respect to the IRS, is given by

$$
\left[u_{i}\right]=\left[T_{i j}\right]\left[u_{j}^{o}\right],
$$

where

$$
\left[T_{i j}\right]=\left[\begin{array}{lll}
1 & -\sin \nu & \sin \eta \\
\sin \nu & 1 & -\sin \xi \\
-\sin \eta & \sin \xi & 1
\end{array}\right],
$$

and $\left[u_{j}^{o}\right]$ is the velocity vector in the misaligned gust probe coordinate system.

We now integrate around the circle. We impose the condition that the measured mean vertical velocity around the circle is zero, that is, $\bar{w}^{o}=0$. Then we have

$$
\begin{aligned}
& \delta \bar{D}=\int_{0}^{2 \pi} v_{\perp} d \Psi=\int_{0}^{2 \pi} v_{\perp}^{o} d \Psi+\sin \nu \int_{0}^{2 \pi} v_{\|}^{o} d \Psi \quad \text { and } \\
& \delta \bar{\zeta}=\int_{0}^{2 \pi} v_{\|} d \Psi=\int_{0}^{2 \pi} v_{\|}^{o} d \Psi-\sin \nu \int_{0}^{2 \pi} v_{\perp}^{o} d \Psi
\end{aligned}
$$

Thus, the error in $\bar{D}$ and $\bar{\zeta}$ introduced by misalignment of the coordinate systems depends only on any residual misalignment between the heading and sideslip measurements; the errors in $\bar{D}$ and $\bar{\zeta}$ are approximately $v$ 


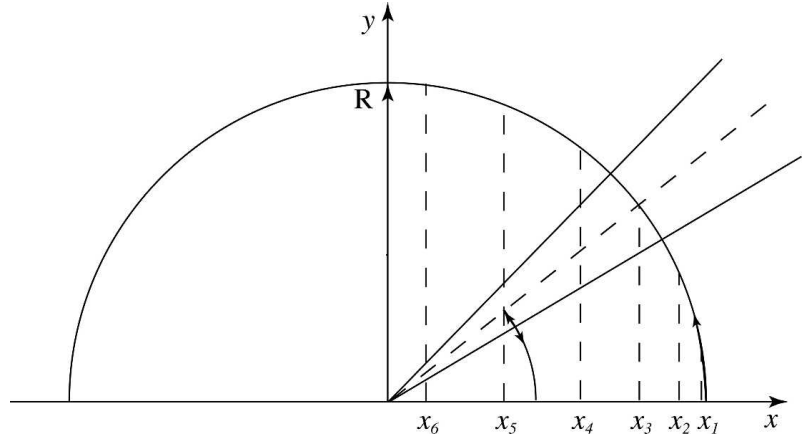

FIG. 4. Schematic of the procedure for estimating the variance of distances $x_{i}$ from the reference line $y=0$ to independent estimates of $u_{i}$ for equally spaced circular segments.

times the measured vorticity and divergence, respectively. This shows that if our calibration procedures discussed above succeed in reducing $v \ll 1$, any remaining contribution of the coordinate system misalignment can be expected to contribute negligible bias to the measured $\bar{D}$ and $\bar{\zeta}$.

\section{b. Random errors}

We base the analysis of random error in the estimates of divergence and vorticity around a circle on the expression for the random error variance of the estimate of the trend (gradient) of a variable given by Bevington (1969). Applying this expression to the $u$ component of the wind field,

$$
\sigma_{\partial u / \partial x}^{2} \simeq \frac{\sigma_{u}^{2}}{N \sigma_{x}^{2}} .
$$

In this expression, $N$ is the number of independent measurements, $\sigma_{u}^{2}$ is the variance of the departure of all the independent wind component estimates $u_{i}$ around the circle from a least squares linear fit, $\tilde{u}_{i}=a+b x_{i}$ :

$$
\sigma_{u}^{2}=\frac{1}{N-2} \sum_{i=1}^{N}\left(u_{i}-a-b x_{i}\right)^{2},
$$

and $\sigma_{x}^{2}$ is the variance of the distances along the $x$ axis from a reference line to each independent measurement of $u_{i}$ (see Fig. 4):

$$
\sigma_{x}^{2}=\frac{1}{N} \sum_{i=1}^{N} x_{i}^{2}-\left(\frac{1}{N} \sum_{i=1}^{N} x_{i}\right)^{2} .
$$

An identical relation holds for $v_{i}$.

As shown in Fig. 4, the separation distance can be written as

$$
x_{i}=R \cos \frac{2 \pi}{N}(i-\delta)
$$

where $i$ is an integer $\leq N$ and $\delta \leq 1$ is a phase angle factor to account for an arbitrary starting point of the circular leg relative to the $x$ axis. Then, substituting (31) into (30), the normalized variance of this separation distance is

$$
\frac{\sigma_{x}^{2}}{R^{2}}=\frac{1}{N}\left[\sum_{i=1}^{N} \cos ^{2} \frac{2 \pi}{N}(i-\delta)-\frac{1}{N}\left(\sum_{i=1}^{N} \cos \frac{2 \pi}{N}(i-\delta)\right)^{2}\right] .
$$

It can easily be shown that as $N$ increases, $\sigma_{x}^{2} / R^{2} \rightarrow 0.5$ regardless of the value of $\delta$. For example, for $N \geq 10$, $\left|0.5-\sigma_{x}^{2} / R^{2}\right| \leq 0.05$. We use the integral scales $\lambda_{u, v}$ to characterize the separation distances along the arc of independent estimates of $u$ and $v$. Because typical values for $\lambda_{u}$ and $\lambda_{v}$ are $<1 \mathrm{~km}$ (Table 1) and the measurements of $u$ and $v$ are obtained along the circle with circumference $2 \pi R \simeq 180 \mathrm{~km}$, the number of independent estimates of $u_{i}$ is $\sim 200$, so that

$$
\frac{\sigma_{x}^{2}}{R^{2}} \simeq 0.5 \text {. }
$$

Substituting $N \simeq 2 \pi R / \lambda_{u}$ and (33) into (28),

$$
\sigma_{\partial u / \partial x}^{2} \simeq \frac{\sigma_{u}^{2} \lambda_{u}}{\pi R^{3}},
$$

and similarly for $\sigma_{\partial v / \partial y}^{2}$. Since we consider here both the $u$ and $v$ fields, we need to estimate the contributions of both $\sigma_{\partial v / \partial x}^{2}$ and $\sigma_{\partial v / \partial y}^{2}$ to the error variances of $\bar{D}$ and $\bar{\zeta}$. We assume that

$$
\sigma_{D}^{2} \simeq \sigma_{\partial u / \partial x}^{2}+\sigma_{\partial v / \partial y}^{2} \simeq \frac{\sigma_{u}^{2} \lambda_{u}+\sigma_{v}^{2} \lambda_{v}}{\pi R^{3}} .
$$

For vorticity, we assume $\sigma_{\partial v / \partial x}^{2} \simeq \sigma_{\partial u / \partial y}^{2}$ and $\sigma_{\partial v / \partial y}^{2} \simeq$ $\sigma_{\partial u / \partial x}^{2}$, so that

$$
\sigma_{\zeta}^{2} \simeq \sigma_{D}^{2}
$$

The integral scales $\lambda_{u, v}$ are defined as

$$
\lambda_{u, v} \equiv \frac{1}{R_{u, v}(0)} \int_{0}^{\infty} R_{u, v}(x) d x,
$$

where the autocorrelation function of $u$ is defined as

$$
R_{u}(x) \equiv \int_{0}^{\infty} u\left(x^{\prime}\right) u\left(x^{\prime}+x\right) d x^{\prime},
$$

and similarly for $R_{v}(x)$. In practice, we found that mesoscale contributions to the wind components can have a large impact on the estimates of $\lambda_{u, v}$. To circumvent this, we fit an exponential function to $R_{u}(x)$ from $R_{u}(x)$ $=1(x=0)$ to $R_{u}(x)=0.5$ by a least squares procedure, similar to Lothon et al. (2006). We then use these estimates of $\lambda_{u}$ and $\lambda_{v}$ to calculate $\sigma_{D}^{2}$ as discussed in the next section. 
TABLE 1. Comparison of the mean divergence $\bar{D}$ obtained from in situ airplane measurements, synoptic data reanalyses [40-yr ECMWF Re-Analysis (ERA-40)], QuikSCAT surface winds (Stevens et al. 2007), and differences between the boundary layer growth and tracer flux entrainment rates as reported by Faloona et al. (2005). Also shown are the std dev of the random error $\sigma_{D}$ for the in situ estimates of the divergence, the integral scale of the horizontal winds (used in the estimate of the random error) obtained from fitting an exponential to the autocorrelation function $R_{u}(x)$ out to $R_{u}(x)=0.5$, and the boundary layer depths (also from Faloona et al. 2005).

\begin{tabular}{|c|c|c|c|c|c|c|c|}
\hline \multirow[b]{2}{*}{ Flight } & \multicolumn{4}{|c|}{ Divergence $\left(10^{-6} \mathrm{~s}^{-1}\right)$} & \multirow[b]{2}{*}{$\sigma_{D}\left(10^{-6} \mathrm{~s}^{-1}\right)$} & \multirow[b]{2}{*}{$\lambda_{u, v}(\mathrm{~m})$} & \multirow[b]{2}{*}{$h(\mathrm{~m})$} \\
\hline & $\mathrm{C} 130$ & ERA-40 & QuikSCAT & Faloona & & & \\
\hline RF01 & 9.7 & $\mathrm{n} / \mathrm{a}$ & 5.1 & 8.3 & 0.96 & 582 & 830 \\
\hline RF02 & 9.9 & 4.4 & 5.1 & 9.1 & 1.00 & 621 & 770 \\
\hline RF03 & 14.8 & 6.3 & 7.1 & 5.5 & 1.04 & 552 & 650 \\
\hline RF04 & -4.5 & 3.2 & 1.8 & 1.7 & 1.12 & 712 & 1070 \\
\hline RF05 & 6.7 & 9.6 & 4.3 & 3.3 & 1.05 & 647 & 910 \\
\hline RF07 & 9.5 & 8.5 & 4.0 & 3.6 & 0.98 & 626 & 810 \\
\hline RF08 & 11.6 & 6.1 & 2.0 & 2.3 & 0.68 & 628 & 620 \\
\hline Avg & 8.2 & 6.5 & 4.2 & 4.8 & 0.98 & 624 & 810 \\
\hline
\end{tabular}

\section{Results}

Here we discuss the results of this analysis applied to a set of seven DYCOMS-II flights conducted in the marine stratocumulus regime off the California coast. The experimental details are discussed by Stevens et al. (2003b). Briefly, a total of six to eight 30-min circles were flown $\mathrm{CW}$ and $\mathrm{CCW}$ at four levels in the PBL on each flight: at $100 \mathrm{~m}$ above the surface, $100 \mathrm{~m}$ below and above cloud base, and $100 \mathrm{~m}$ below the PBL top.

The wind data used here have been modified from what was used in earlier analyses (Stevens et al. 2007) by incorporating a correction to the static pressure based on a more accurate in-flight calibration using a trailing cone pressure calibration technique (Brown 1988). The primary motivation for this recalibration was the discovery that momentum flux measurements were slightly heading dependent. The recalibrated wind data considerably reduced this dependency and also resulted in considerably different values of $\bar{D}$ than earlier analyses. Table 1 shows a mean $\bar{D}$ over all the flights of $8.2 \times 10^{-6} \mathrm{~s}^{-1}$, while $\bar{D}$ with the earlier calibration was $-7.0 \times 10^{-6} \mathrm{~s}^{-1}$, which is clearly unreasonable for this climatic regime. This difference points out the sensitivity of $\bar{D}$ to the calibration of the wind-sensing instruments and to uncorrected flow distortion effects. In contrast, $\bar{\zeta}$ is less sensitive to the calibration change, $\bar{\zeta}=-13.3 \mathrm{~s}^{-1}$ for the older calibration compared to $-12.1 \mathrm{~s}^{-1}$ for the value obtained here.

Figure 5 shows $\bar{D}$ and $\bar{\zeta}$ averaged over all levels for each of the seven cases. From the figure we note that the estimated random error due to limited sampling, $\sigma_{D}$, [as shown by the thick vertical bars and calculated using (35)] is significantly smaller than the measured standard deviation of the estimates (shown by the thin vertical bars and calculated from the data after removing a bias from $\beta$ by equating $\bar{D}$ from $\mathrm{CW}$ and $\mathrm{CCW}$ circles), suggesting that our measurements are not limited by the effects of small-scale turbulent wind fluctuations but by real day-to-day and level-to-level variability in $\bar{D}$ and $\bar{\zeta}$. That is, the standard deviation of the estimates for each flight is an overly conservative measure of the random error since the estimates include real temporal and spatial variability. The difference between these two estimates is a measure of this variability. From flight to flight $\sigma_{D}^{2}$ varies little: $\sigma_{D} \simeq \sigma_{\zeta} \simeq 1.0$ $\times 10^{-6} \mathrm{~s}^{-1}$. The observed standard deviation of the estimates is $\simeq 4-8 \times 10^{-6} \mathrm{~s}^{-1}$. With the exception of the divergence estimate of RF04, the estimates of $\bar{D}$ and $\bar{\zeta}$ are commensurate with expectations based on climatology indicative of low Rossby number flow and significant divergence. Note that the mean divergence in Table 1 of $8.2 \times 10^{-6} \mathrm{~s}^{-1}$ (which is the average over the flights in Table 1) over a boundary layer of depth $810 \mathrm{~m}$ corresponds to a subsidence rate at the top of the layer of about $6.6 \mathrm{~mm} \mathrm{~s}^{-1}$ (somewhat larger than observed during RF01; Stevens et al. 2003a). For a mean stratification of $5.9 \mathrm{~K} \mathrm{~m}^{-1}$ (Stevens et al. 2007), this requires
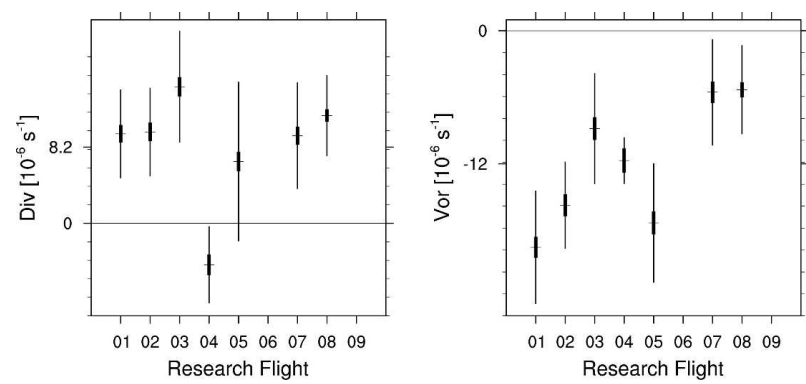

FIG. 5. (left) Divergence and (right) vorticity for seven DYCOMS-II cases. The thick vertical bar is \pm 1 std dev of the estimated random error and the thin vertical bar is \pm 1 std dev of the mean calculated from the measurements. The measured std devs are calculated after removing a bias from $\beta$ by equating $\bar{D}$ from $\mathrm{CW}$ and $\mathrm{CCW}$ circles. 
a mean cooling rate of just over $3 \mathrm{~K} \mathrm{day}^{-1}$ to balance the mean subsidence, which again is somewhat larger than expected based on radiative transfer calculations.

The sampling error obtained from (35) can be compared to that obtained by Lenschow et al. (1999) using the line-integral method (3). The line-integral equivalent to $(35)$ is

$$
\sigma_{D}^{2}(\text { line-integral }) \simeq \frac{4 \sigma_{\perp}^{2} \lambda_{\perp}}{\pi R^{3}}
$$

which leads to a random error standard deviation of roughly 4-6 times that from (35). This is due to $\lambda_{\perp} \simeq$ $2-3 \times \lambda_{u, v}$ and to the larger numerical factor in (39). Thus, while the line-integral method has a larger sampling error, and requires a stationary wind field and closed flight track, it does have the advantage over the regression method of not requiring an assumption about the spatial structure of the wind field.

Looking closer at the RF04 data, we find little evidence that this flight was anomalous in quantities other than the estimated divergence, which was $-4.5 \times 10^{-6}$ $\mathrm{s}^{-1}$. The standard deviation of $\bar{D}$ is not larger on RF04 as compared to other flights-mean convergence was robustly measured among each of its circles. Furthermore, $\bar{\zeta}$ on RF04 is not anomalous. RF04 did tend to have a deeper boundary layer than was observed on other days, and the boundary layer deepened more rapidly than was observed on other flights, but in both respects it was not that different from $\mathrm{RF} 05$, for which the divergence was measured to be $6.7 \times 10^{-6} \mathrm{~s}^{-1}$. Both the thermodynamic quantities and the evolution of the flight tracks suggest that the flight pattern tracked the Lagrangian evolution of the air mass at least as well as the other flights. Similarly, although the winds were especially light on RF04, their vertical and temporal consistency was at least as good as was observed on the other flights. Therefore, in the absence of other explanations we consider the possibility that the measured convergence during RF04 is real.

To the extent this measurement reflects the mean convergence through the boundary layer (which it should because it is estimated from circles well distributed throughout the PBL) it implies a vertical velocity of about $0.48 \mathrm{~cm} \mathrm{~s}^{-1}$ at the top of the layer, which would imply that, in the absence of entrainment, the layer should have deepened by about $100 \mathrm{~m}$ over the 6-h period on station. Lidar observations of cloud-top height from the circular legs flown above the boundary layer both near the beginning and end of the time on station suggest that the layer deepened by somewhat less than $150 \mathrm{~m}$. Thus, the observed rate of PBL deepening is enough to account for the observed conver- gence, but the resulting entrainment rate is small. This raises the possibility that, at least in this case, there may be some error that has not been accounted for. Candidates include unquantified flow distortion effects, nonlinear spatial or temporal variations in the mean wind field, or withdrawal of the lidar-sensed cloud top from the top of the PBL.

Table 1 shows a comparison of our in situ estimates of $\bar{D}$ with a variety of other estimates from the literature. The estimates by the European Centre for Medium-Range Weather Forecasts reanalysis and from the SeaWinds measurement on Quick Scatterometer (QuikSCAT) are taken from Stevens et al. (2007) and measure the divergence of the 10 -m winds over an area somewhat larger than an individual circle. Both are averaged over a period of $12 \mathrm{~h}$ centered at the middle of the flight period. For QuikSCAT this corresponds to no more than two overpasses and in some cases (e.g., RF01) none. The estimates of $\bar{D}$ by Faloona et al. (2005) are made by taking the difference between the entrainment rates and the rate at which the boundary layer deepens to derive a net rate of subsidence at cloud top. The mean divergence is then just the subsidence velocity divided by the boundary layer height. For these estimates, the entrainment is measured by the ratio of the flux of conserved tracers at the top of the PBL to the change in tracer concentration across the PBL, while the evolution of the PBL depth is measured by the change in height of cloud top as measured by a downward-looking lidar during circular flight legs, once near the beginning and again near the end of the time on station.

From Table 1 we note that there are considerable differences in estimates of $\bar{D}$ on a day-to-day basis, and when averaged over all cases, the aircraft estimates seem somewhat high. The lack of correlation on a dayto-day basis is a little disappointing, but perhaps not surprising, given the magnitude of the signal and the differences in the way it is estimated. While all the estimates for RF04 give the smallest divergence rate of all the flights, only the in situ estimate suggests that the flow is converging. Based on these data it is difficult to rule out any of the methods for determining $\bar{D}$, especially given that no attempt has been made to quantify sources of uncertainty or bias in the previously published estimates. We believe that the largest source of unquantified uncertainty in our estimates of $\bar{D}$ is associated with flow distortion effects and limitations associated with inflight calibrations. This is borne out by the large difference of about $-15 \pm 1 \times 10^{-6} \mathrm{~s}^{-1}$ between $\bar{D}$ using the newly calibrated data and $\bar{D}$ from the previous data. The small standard deviation of $\pm 1 \times$ 
$10^{-6} \mathrm{~s}^{-1}$ indicates that the major effect of these errors is a bias in the measured $\bar{D}$.

Since our quantified errors indicate that $\bar{D}$ can be measured to an accuracy per flight of $\sim \pm 1 \times 10^{-6} \mathrm{~s}^{-1}$ with accurate air velocity measurements, this suggests that the development of measurement systems that do not suffer from such effects (e.g., a Doppler laser system) offers the potential of yielding estimates of $\bar{D}$ from the in situ technique that is more accurate than any other current method, and thus offers an argument for the development of such a system.

\section{Summary and conclusions}

We have described here a technique for estimating divergence from airplane measurements of the mean wind field obtained from circular flight paths. The method, based on simple linear regression to estimate spatial and temporal gradients in the wind field, appears to be more robust, and less sensitive to sampling error, than previously proposed methods. The extreme accuracy required for this measurement combined with the use of a circular flight path uncovered a systematic error in airplane wind measurements heretofore undocumented. Essentially, it is a result of a mean roll angle, incurred by continuously turning the aircraft, multiplied by an offset in the attack angle that does not change sign when the aircraft flies a circle in the opposite direction. We describe a way to eliminate this error, which we argue should be incorporated as the standard method for calibrating the wind field measurement system for airborne platforms. We then compare the mean divergence calculated using the aircraft-based wind estimates with a number of other estimates of divergence for the same flights. The mean aircraft divergence is within expectations, although a bit larger than the other estimates. Furthermore, there are significant differences from case to case that are difficult to explain but are indicative of the limitations of the various methods.

Given the extent to which estimates of the mean divergence based on the wind field measurements are commensurate with both expectations and other methods, it seems plausible that a measurement system that addresses the main uncertainties in the current method, namely, unquantified flow distortion effects and inaccuracies associated with in-flight calibrations, would make it possible to routinely estimate divergence to much greater accuracy than is currently possible using existing methods. This would be a significant step forward in airborne measurement science.

Acknowledgments. Any opinions, findings, and conclusions or recommendations expressed in this publication are those of the authors and do not necessarily reflect the views of the National Science Foundation. The work of V. S. J. and B. S. was supported by NSF Grants ATM-0336849 and ATM-0097053 and NASA Grant NAG512559. We appreciate the support of the NCAR Research Aviation Facility in providing improved datasets and assisting in elucidating errors and limitations in the current C-130 air motion sensing system. We thank John Kalogiros, Marie Lothon, and the two anonymous reviewers for their helpful comments.

\section{REFERENCES}

Angevine, W. M., 1997: Errors in mean vertical velocities measured by boundary layer wind profilers. J. Atmos. Oceanic Technol., 14, 565-569.

Bevington, P., 1969: Data Reduction and Error Analysis for the Physical Sciences. McGraw-Hill, 336 pp.

Brost, R. A., D. H. Lenschow, and J. C. Wyngaard, 1982: Marine stratocumulus layers. Part I: Mean conditions. J. Atmos. Sci., 39, 800-817.

Brown, E. N., 1988: Position error calibration of a pressure survey aircraft using a trailing cone. NCAR Tech. Note NCAR/TN$313+$ STR, 29 pp. [Available online at http://www.library. ucar.edu/uhtbin/hyperion-image/DR000579.]

Davies-Jones, R., 1993: Useful formulas for computing divergence, vorticity, and their errors from three or more stations. Mon. Wea. Rev., 121, 713-725.

Faloona, I., and Coauthors, 2005: Observations of entrainment in eastern Pacific marine stratocumulus using three conserved scalars. J. Atmos. Sci., 62, 3268-3285.

Gultepe, I., A. J. Heymsfield, and D. H. Lenschow, 1990: A comparison of vertical velocity in cirrus obtained from aircraft and lidar divergence measurements during FIRE. J. Atmos. Oceanic Technol., 7, 58-67.

Kalogiros, J. A., and Q. Wang, 2002: Calibration of a radomedifferential GPS system on a Twin Otter research aircraft for turbulence measurements. J. Atmos. Oceanic Technol., 19, 159-171.

Lenschow, D. H., 1986: Aircraft measurements in the boundary layer. Probing the Atmospheric Boundary Layer, D. H. Lenschow, Ed., Amer. Meteor. Soc., 39-55.

_ P. B. Krummel, and S. T. Siems, 1999: Measuring entrainment, divergence, and vorticity on the mesoscale from aircraft. J. Atmos. Oceanic Technol., 16, 1384-1400.

Lothon, M., D. H. Lenschow, and S. D. Mayor, 2006: Coherence and scale of vertical velocity in the convective boundary layer from a Doppler lidar. Bound.-Layer Meteor., 121, 521-536.

Mapes, B. E., and R. A. Houze, 1995: Diabatic divergence profiles in western Pacific mesoscale convective systems. J. Atmos. Sci., 52, 1807-1828.

_ wind divergence in regions of tropical convection. Mon. Wea. Rev., 133, 1808-1824.

Stevens, B., and Coauthors, 2003a: On entrainment rates in nocturnal marine stratocumulus. Quart. J. Roy. Meteor. Soc., 129, 3469-3493.

$\ldots$, and $-2003 \mathrm{~b}$ : Dynamics and chemistry of marine stratocumulus-DYCOMS-II. Bull. Amer. Meteor. Soc., 84, 579-593.

— A. Beljaars, S. Bordoni, C. Holloway, M. Köhler, S. Krueger, V. Savic-Jovcic, and Y. Zhang, 2007: On the structure of the lower troposphere in the summertime stratocumulus regime of the northeast Pacific. Mon. Wea. Rev., 135, 985-1005. 